\title{
GSM BASED PATIENT MONITORING SYSTEM IN NICU
}

\author{
Bhargavi Sundara $^{1}$, Dr. Kalyana Chakravarthi Sarvepalli ${ }^{2}$, Sri Harsha Davuluri ${ }^{3}$ \\ ${ }^{1,3}$ Department of Electronics and Communication Engineering, MRECW, Hyderabad \\ bhargavisundara@gmail.com,doctorkc1984@gmail.com, harshadavuluri@gmail.com
}

\begin{abstract}
The project concentrates a selective section of patient monitoring: neonatal care. Several patient monitors are available in the market. Yet it is quite a daunting task in countries with high population like India, because of insufficient resource space availability. It is difficult to keep a track of several patients at a time from both inside and outside hospital premises/medical facility, which includes remote locations. The project involves with sensing the vitals of the newly born babies which are very different when compared to adult patients and transmit these signals to the computer monitor at the nursing station. Also based on the preset threshold values for the recorded values, an alarm system will be introduced with an immediate transfer of the corresponding patient data to the registered medical professional. This paper attempts to design and implement patient monitoring and real time feedback mechanism, equipped with wireless transmission via GSM and Zigbee. Using Zigbee for in-house monitoring and GSM for external correspondence is proposed. The proposed system is likely to be efficient, economical, easy to use, and portable, have wide application potential due to flexibility in the design and software.
\end{abstract}

Keywords: Bio signal Processing, Neonatal, Wireless health monitoring, zigbee, GSM, Wifi, Portable multi parameter health monitor. - ***

\section{INTRODUCTION}

\section{A. Health monitoring device}

The development of Health Monitor with multi parameter capability for neonatal applications has been motivated mainly by increasing healthcare costs and technological advances in miniature bio-sensing devices, microelectronics and wireless communication techniques [1].

This system can comprise various types of small physiological sensors, which enable continuous monitoring of a variety of physiological parameters such as

Airflow control of patient

- Body temperature data.

- Galvanic skin response measurements.

- Body position detection.

- Monitoring ECG signal.

- Pulse and oxygen functions.

- Multiple data visualization systems.

Furthermore, due to embedded transmission modules and processing capabilities health monitoring system can facilitate low-cost solutions for continuous monitoring. [2], [3], [4]

In the present paper, the parameters of Pulse and Oxygen in blood, ECG and Airflow sensors incorporations are discussed.

\section{WORK}

A. Pulse and Oxygen in Blood (SPO2)

\section{1). Connecting the sensor}

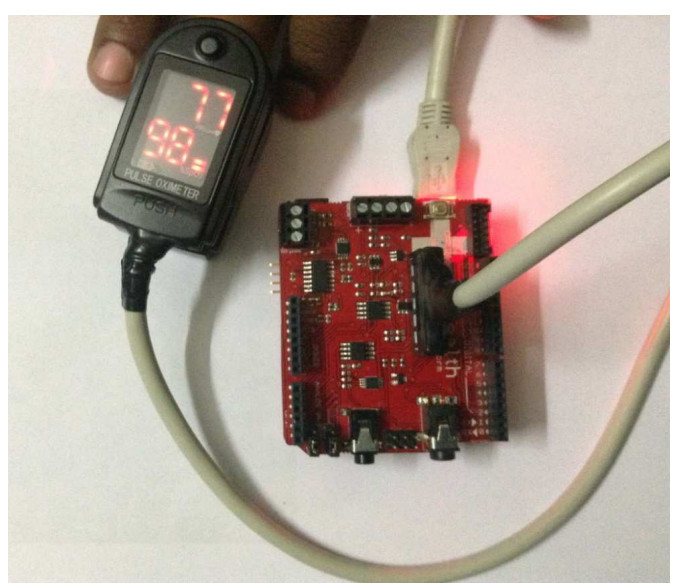

Figure1: Pulse oximeter sensor connected to Medical diagnostic platform

Connect the module in the Medical diagnostic platform as shown in figure 1 . The sensor has only one way of connection to prevent errors and make the connection easier.

Once a finger is inserted into the sensor and on button is pressed, one will get the values in the sensor screen after few 
seconds. After this, attach the interruptions in the code to get the data from the sensor. The sensor will interrupt the process to refresh the data stored in private variables.

The digital pin 6 of Arduino is the pin where sensor sends the interruption and the function read Pulseoximeter will be executed. Also one must configure some basic parameters, to start the communication between the Arduino/RasberryPi and sensor.

\section{2). Aquiring data}

a) Mobile app showing $\mathrm{SPO} 2$ readings

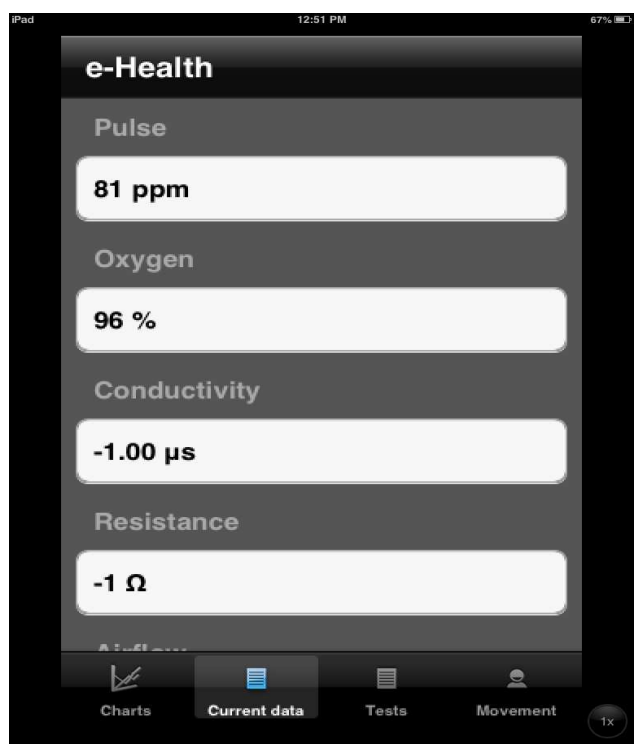

Figure2 Iphone app showing SPO2 output

\section{b). Serial monitoring of SPO2 readings}

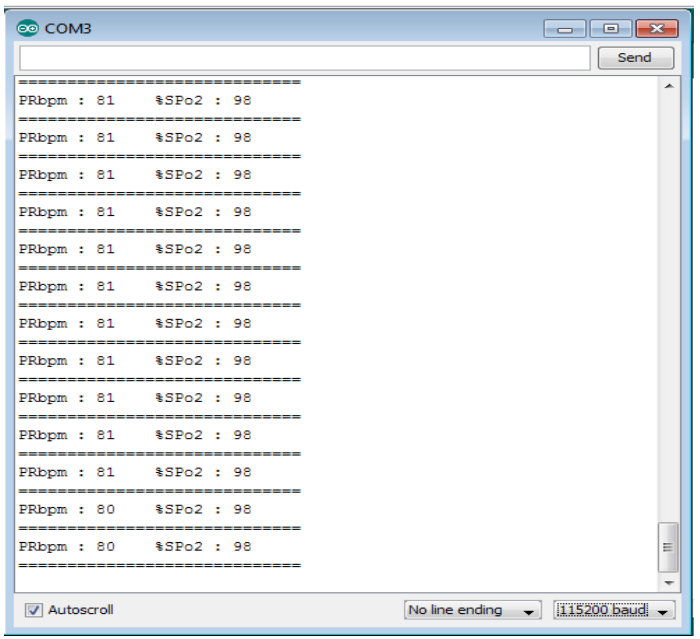

The App shows the information the nodes are sending which contains the sensor data gathered the SPO2 outputs is shown in figure 2 and figure 3.

\section{B. Electrocardiogram (ECG)}

\section{1). Connecting the sensor}

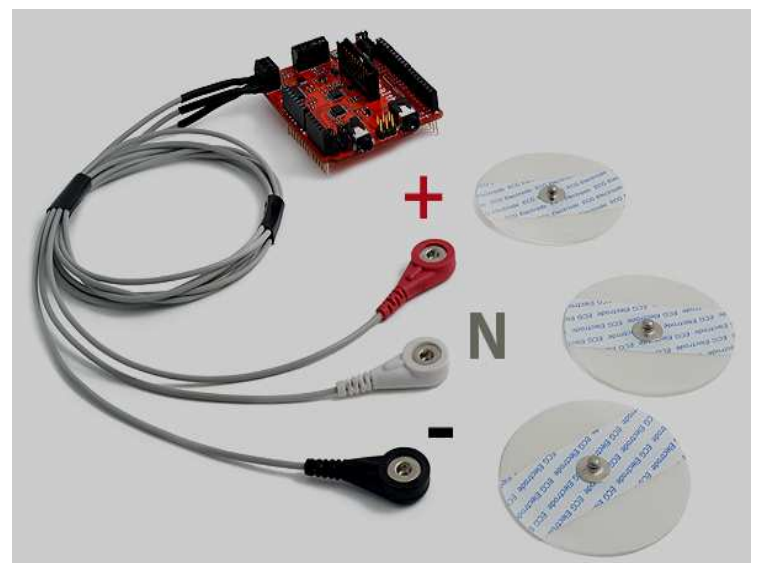

Figure4: ECG leads connected to Medical diagnostic platform

The ECG leads with disposable electrodes are shown fig.4. Connect the ECG lead to the electrodes.

\section{2). Acquiring data}

\section{a).Mobile app showing ECG graph}

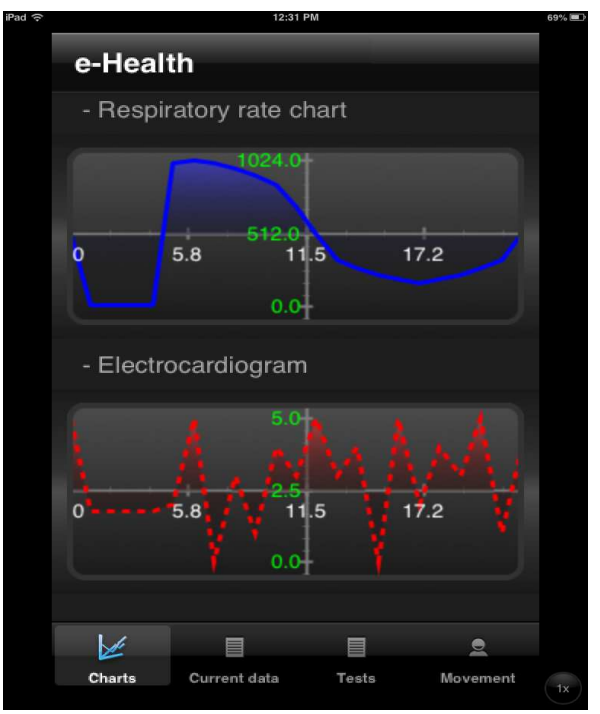

Figure5: Iphone app displaying ECG waveform of patient

Figure3: SPO2 readings on Arduino serial display 


\section{b). Continuous serial monitoring in KST}

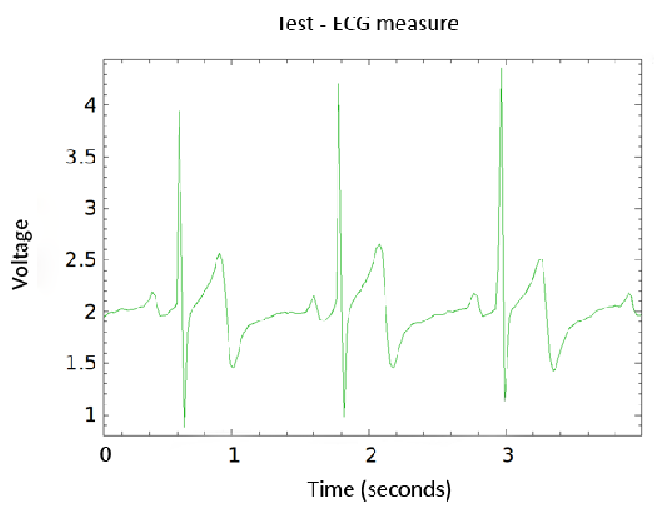

Figure6: Patient ECG waveform on KST serial monitor

The App shows the information the nodes are sending which contains the sensor data gathered the ECG output shows in figure 5 and figure 6 .

\section{Airflow: breathing}

\section{1). Connecting the sensor}

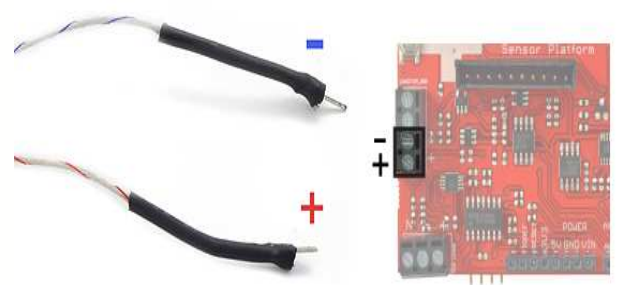

Figure7: Connecting Airflow sensor to Medical diagnostic platform

Connect the red wire with the positive terminal (marked as "+" in the board) and the black wire with the negative terminal (marked as "-" in the board). After connecting the cables, tighten the screws the airflow outputs can shown in figures

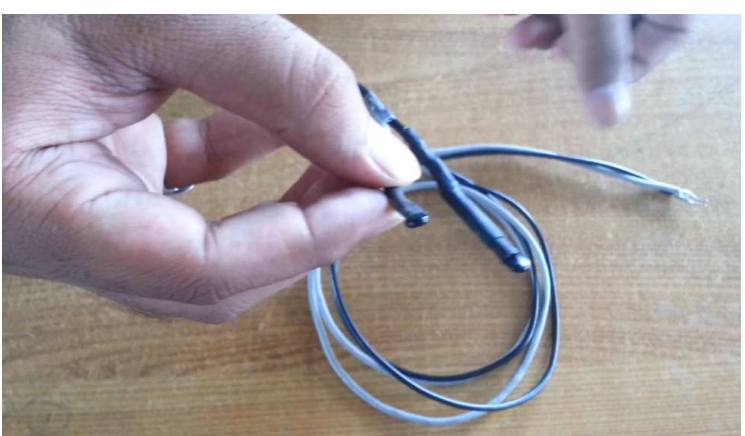

Figure8: Airflow sensor
In the Medical diagnostic platform, Airflow sensor has two connections (positive and negative) the Connect Airflow sensor to Medical diagnostic platform as shown in figure 7 and figure 8 .

\section{2). Aquiring Data}

\section{a). Continuous serial monitoring in KST}

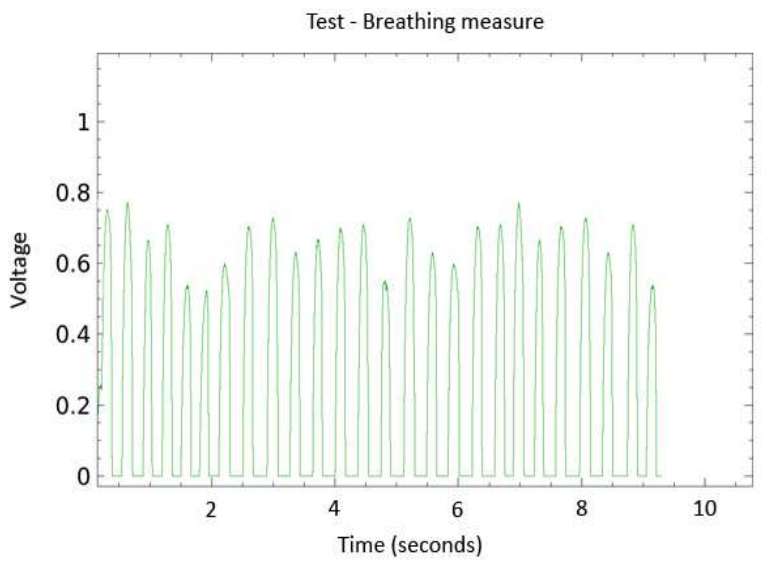

Figure9: Respiratory graph in KST serial monitor

\section{b). Continuous monitoring in Arduino}

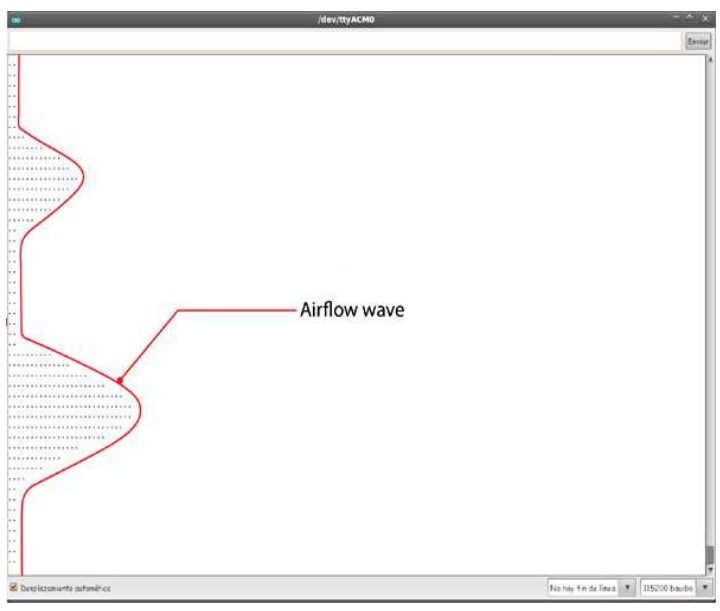

Figure10: Arduino serial monitor showing respiratory rate wave

Airflow sensor monitor display in KST and Arduino is shown in figure 9 and figure 10. 


\section{c). Mobile App showing airflow graph}

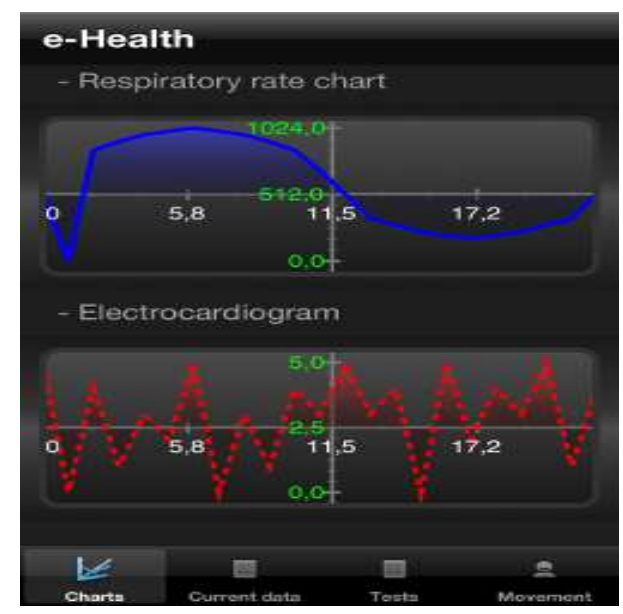

Figure11: Iphone app showing respiratory rate graph

The App shows the information the nodes are sending which contains the sensor data gathered the respiratory rate graph in Iphone app is shown in fig 11.

\section{CONCLUSIONS}

The developed prototype platform utilizes low cost/ economic components and constitutes a novel paradigm of how multiple wireless-enabled biosensors can be utilized for electronic health monitoring applications. The developed interfaces on both the smart-phone and the remote workstation allow the user to have a complete picture of the patient's health and to have instant access to real-time and past physiological data.

\section{REFERENCES}

[1] T. G, "The Agenda of Wearable Healthcare," IMIA Year book of Medical Informatics, pp. 125-138, 2005.

[2] G. L and I. I, "Wearable and Portable ehealth systems," IEEE Engineering in Medicine and Biology, vol. 26, pp. 29-33, 2007.

[3] P. A and B. N, "A Survey on Wearable Biosensor Systems for Health Monitoring," in 30th Annual International Conference IEEE EMBS, 2008.

[4] P. A and B. N, "Prognosis-A Wearable Health Monitoring System for People at Risk: Methodology and Modeling," IEEE Transactions of Information Technology in Biomedicine, vol. 14, no. 3, pp. 613-621, 2010. 